\title{
Attempts to Classify Herbicola Group-Enterobacter agglomerans Strains by Deoxyribonucleic Acid Hybridization and Phenotypic Tests
}

\author{
DON J. BRENNER, ${ }^{1 *}$ G. RICHARD FANNING ${ }^{2}$ JEAN K. LEETE KNUTSON,${ }^{1}$ ARNOLD G. STEIGERWALT ${ }^{1}$
} AND MICAH I. KRICHEVSKY ${ }^{3}$

Molecular Biology Laboratory, Division of Bacterial Diseases, Center for Infectious Diseases, Centers for Disease Control, Atlanta, Georgia $30333^{1}$; Division of Biochemistry, Walter Reed Army Institute of Research, Washington, D.C. $20012^{2}$; and Microbial Systematics Section, National Institute of Dental Research, Bethesda, Maryland $20205^{3}$

\begin{abstract}
There are seven names on the Approved Lists of Bacterial Names that have been treated as partial or total synonyms for strains belonging to the Enterobacter agglomerans-Herbicola group of Erwinia species complex. A total of 124 strains belonging to this complex, isolated mainly from plant and human sources, were studied by deoxyribonucleic acid relatedness and by a variety of biochemical tests. Ninety of these strains fell into 13 deoxyribonucleic acid hybridization groups ( 2 to 13 strains per group), and the remaining 34 strains did not fit into any hybridization group. Nine of the hybridization groups could be separated biochemically, whereas four hybridization groups could not. Our results point out the inadequacy of the classification schemes presently used for these organisms, the inadequacy of the present nomenclature, the extreme diversity of the strains presently classified in the Enterobacter agglomerans-Herbicola group of Erwinia species complex, and the need for additional, in-depth studies of these organisms.
\end{abstract}

Strains belonging to the Herbicola group of Erwinia species-Enterobacter agglomerans complex (Herbicola-Agglomerans group) were described as early as $1888(2,14)$. The Herbicola-Agglomerans group contains organisms that are found as pathogens or saprophytes on plants $(12,18,21)$, in soil and water $(18,22)$, in at least one insect $(23)$, in animals (18), and as pathogens or secondary invaders in a variety of human infections $(5,24,27)$. The name first given to these strains apparently was "Bacterium agglomerans" $(2,14)$; subsequently, they have been placed in 13 different genera under 27 species names, resulting in a total of at least 56 different nomenclatural combinations (Table 1). Studies by Graham et al. $(6,18,19)$ and Dye $(8-12)$ resolved much of the nomenclatural confusion surrounding the HerbicolaAgglomerans group. Largely as a result of the efforts of these workers, there are now only seven species names to consider.

In Bergey's Manual of Determinative Bacteriology, 8th ed. (23), three species, one of which has two varieties, are listed in the Herbicola group of Erwinia species; these are Erwinia herbicola (with the varieties Erwinia herbicola var. herbicola and Erwinia herbicola var. ananas), Erwinia stewartii, and Erwinia uredovora. After comparing human and plant isolates, Ewing and Fife $(13,14)$ proposed the name Enterobacter agglomerans for the Herbicola-Agglomerans group. These authors described seven anaerogenic and four aerogenic biogroups of Enterobacter agglomerans, which they concluded was a senior subjective synonym for the three Erwinia species in the Herbicola group. The three Erwinia species and Enterobacter agglomerans are on the Approved Lists of Bacterial Names (28). Three additional species on the Approved Lists are also thought to belong to the Herbicola-Agglomerans group. These are Escherichia adecarboxylata, Erwinia ananas (as a species rather than as a variety of Erwinia herbicola), and Erwinia milletiae.

Through 1965 there were only three reports that the Herbicola-Agglomerans group was associated with human

\footnotetext{
* Corresponding author.
}

disease $(6,7,25,27)$. Only a few human isolates were included in the studies of the Herbicola-Agglomerans group done by plant and agricultural bacteriologists. Reports of Herbicola-Agglomerans group isolates from humans increased beginning in the late 1960s. Biochemical characterization of groups of human strains was first reported by von Graevenitz $(32,33)$ and Bottone et al. $(3,17)$.

Interest in these organisms peaked in 1971, when they were implicated in a nationwide nosocomial septicemia outbreak due to contaminated intravenous products in which 40 of 378 patients died (24). Ewing and Fife studied the outbreak strains, compared them with the Herbicola group of Erwinia, and concluded that the two sets of strains belonged to the same species $(13,14)$. Reports of clinical isolates belonging to the Herbicola-Agglomerans group and of biochemical characterization of these isolates subsequently appeared from workers in many countries $(5,16,26,27$, 31).

The purpose of this study was to determine whether any or all of the Herbicola group of Erwinia species and Enterobacter agglomerans comprise a single species or whether these taxa represent more than one species.

(Some of the results were taken from a thesis presented by J.K.L.K. to the University of North Carolina, Chapel Hill.)

\section{MATERIALS AND METHODS}

Nomenclature. Erwinia herbicola, Erwinia stewartii, Erwinia uredovora, Erwinia ananas, Erwinia milletiae, Escherichia adecarboxylata, and Enterobacter agglomerans all appear on the Approved Lists (28). There seems to be agreement that each of these names represents one or more species in the same group, but disagreement as to the number of species in this group and as to which, if any, of these names are synonyms. We use the term HerbicolaAgglomerans group to refer to all of the organisms in this group. Species names were used to denote the names under which specific strains were received and to compare various taxonomic proposals for these organisms. Names not on the Approved Lists are written in quotation marks (Table 1). 
TABLE 1. Presumed synonyms for members of the HerbicolaAgglomerans group

\begin{tabular}{|c|c|}
\hline Name & Reference $(s)^{a}$ \\
\hline Escherichia adecarboxylata ................ & 22 \\
\hline Enterobacter agglomerans $\ldots \ldots \ldots \ldots \ldots \ldots \ldots$ & 14 \\
\hline "Bacterium agglomerans" $\ldots \ldots \ldots \ldots \ldots \ldots \ldots$ & 14 \\
\hline "Bacillus ananas" $\ldots \ldots \ldots \ldots \ldots \ldots \ldots \ldots \ldots \ldots \ldots \ldots \ldots$ & 12 \\
\hline "Bacterium ananas" $\ldots \ldots \ldots \ldots \ldots \ldots \ldots \ldots$ & 23 \\
\hline 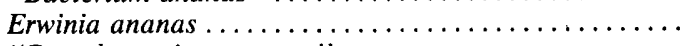 & 12 \\
\hline "Pectobacterium ananas" $\ldots \ldots \ldots \ldots \ldots \ldots \ldots$ & 23 \\
\hline "Erwinia herbicola var. ananas" ............. & 12 \\
\hline "Xanthomonas annamalaiensis" ............ & 11,12 \\
\hline "Xanthomonas balsamivorum" .............. & 11,12 \\
\hline 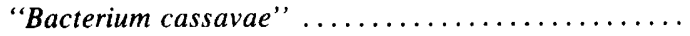 & 12 \\
\hline 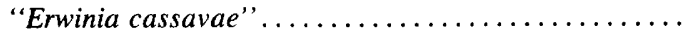 & 12,20 \\
\hline "Bacillus citrimaculans". . & 12,20 \\
\hline 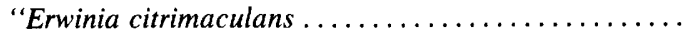 & 12,20 \\
\hline "Xanthomonas cosmosicola" .............. & 11,12 \\
\hline 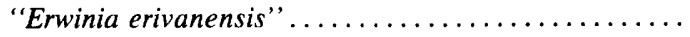 & 12 \\
\hline "Xanthomonas esculenti" ......... & 11,12 \\
\hline "Bacillus flavidus" . . . . . . . . . . . . . . . . & 12 \\
\hline 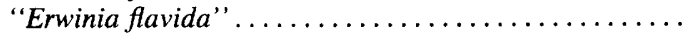 & 12 \\
\hline "Agrobacterium gypsophilae"...... & 19 \\
\hline "Bacterium herbicola" ........... & 10,12 \\
\hline "Bacterium herbicola aureum". ............. & 10,12 \\
\hline Erwinia herbicola . . . . . . . . . . . . . . . & 12 \\
\hline "Erwinia mangiferae"”... & 12,20 \\
\hline 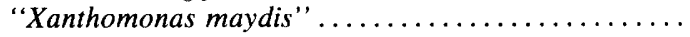 & 11,12 \\
\hline "Bacillus milletiae" ..... & 12 \\
\hline Erwinia milletiae . . . . . . . . . . . . . . & 12,20 \\
\hline 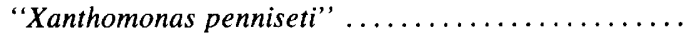 & 11,12 \\
\hline "Flavobacterium rhenanum" ................ & 23 \\
\hline "Xanthomonas rubrisorghi" ............. & 11,12 \\
\hline "Xanthomonas tagetis" $\ldots \ldots \ldots \ldots \ldots \ldots \ldots \ldots$ & 11,12 \\
\hline "Flavobacterium trifolii" ................... & 10,12 \\
\hline "Flavobacterium trifolium" ................ & 10,12 \\
\hline "Pseudomonas trifolii".... & 10,12 \\
\hline 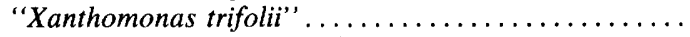 & 10,12 \\
\hline "Chromobacterium typhi-flavum" ............ & 12 \\
\hline "Bacterium typhi flavum" ....... & 12 \\
\hline "Bacillus vitavorus" ...... & 12 \\
\hline "Erwinia vitavora" $\ldots .$. & 12,20 \\
\hline "Aplanobacter stewarti". & 9 \\
\hline "Bacillus stewarti" ... & 9 \\
\hline "Bacterium stewarti". & 9 \\
\hline Erwinia stewartii...... & 9,12 \\
\hline "Pseudobacterium stewarti" ................. & 23 \\
\hline "Pseudomonas stewarti". & 9,12 \\
\hline "Flavobacterium herbicola". & 10,12 \\
\hline "Pseudomonas herbicola". . & 12 \\
\hline "Xanthomonas herbicola". & 18 \\
\hline "Xanthomonas indica". & 11,12 \\
\hline "Bacillus lathyri"'. & 12 \\
\hline "Erwinia lathyri"' & 10,12 \\
\hline "Bacillus mangiferae". & 12 \\
\hline "Phytobacterium stewartii". & 9 \\
\hline "Xanthomonas stewarti". & 9 \\
\hline Erwinia uredovora ....... & 8,12 \\
\hline "Xanthomonas uredovorus" .......... & 8,12 \\
\hline
\end{tabular}

${ }^{a}$ Reference(s) in which the name was proposed or in which the original work is cited.

Strains. The bacterial strains received as members of the Herbicola-Agglomerans group are listed in Table 2. The strains of other members of the Enterobacteriaceae from which deoxyribonucleic acid (DNA) was prepared have been listed previously (30).

DNA relatedness. The methods used to prepare DNA labeled with ${ }^{32} \mathrm{P}$ and unlabeled DNA, the methods used in the hydroxyapatite procedure for DNA hybridization, and the method used for calculating DNA relatedness have been described previously (4).

Biochemical tests. The conventional biochemical test media and conditions used have been described previously (21). The API 20E biochemical kit system (Analytab Products, Inc.) was used according to the directions of the manufacturer, except that incubations were done at both $25 \pm 1$ and 36 $\pm 1^{\circ} \mathrm{C}$. The API 50 research system (API 50R; Analytab Products, Inc.) was used according to the instructions of the manufacturer, with the following exceptions. Incubations were done at $36 \pm 1^{\circ} \mathrm{C}$. A conventional methyl red test was done in parallel with the API 50 methyl red test; both were read after $48 \mathrm{~h}$, and the results of the conventional reaction were used if the results differed. All other tests were read after 24 and $48 \mathrm{~h}$. Reactions were graded on a scale from 0 to 5 depending on the intensity of the color produced, as directed by the manufacturer; on this scale a score of 0 was negative, 1 was weak or doubtful, 2 was doubtful positive, 3 was weakly positive, 4 was positive, and 5 was strongly positive. A reaction with a score of 2 was arbitrarily deemed negative, and a reaction with a score of 3 was arbitrarily deemed positive.

Computer-assisted phenetic analysis. All computations were performed by using the programs described by Walczak and Krichevsky (34-36). Interstrain similarities generated from 44 conventional biochemical tests were calculated by using both the simple matching and Jaccard coefficients. Clustering was done by using both single linkage and unweighted average linkage (29).

Antimicrobial agent susceptibility tests. Antibiograms were done on Mueller-Hinton agar by the disk method of Bauer et al. (1), as previously described (15).

\section{RESULTS}

We determined the level of DNA relatedness of 124 strains of the Herbicola-Agglomerans group to each of 17 reference strains chosen from this group. The reference strains were chosen arbitrarily, and their DNAs were labeled with ${ }^{32} \mathrm{P}$. We also determined the level of relatedness of each reference strain to representative species of the Enterobacteriaceae. At least four strains from each of the 11 biogroups of Enterobacter agglomerans (14) were included in the study, as were strains received as Erwinia herbicola, Erwinia ananas, Erwinia stewartii, Erwinia uredovora, Erwinia milletiae, "Erwinia mangiferae," "Erwinia lathyri," "Erwinia cassavae," "Erwinia maydis," and "Xanthomonas trifolii"' (Table 2).

A total of 13 DNA hybridization groups (groups of strains whose DNAs were $70 \%$ or more related) containing from 2 to 13 strains each were identified from the DNA hybridization reactions (Tables 2 and 3). Altogether, 90 strains were classified into these DNA hybridization groups, whereas the remaining 34 strains did not belong to any group (Table 2). DNAs from four of these ungrouped strains (strains 486-51, 4908-71, ATCC 23372, and ICPB SS 11) were labeled, but they showed less than species level relatedness to unlabeled DNAs from the remaining ungrouped strains and to representative DNAs from each of the 13 previously established groups (data not shown). All of the hybridization groups were unique at the species level; no strain showed $70 \%$ or greater relatedness to strains representing more than one hybridization group. The levels of relatedness between pairs of the 13 hybridization groups ranged from 17 to $64 \%$ (Table 4). Hybridization groups I through VI, VIII, and XIII were $40 \%$ or more interrelated, as were hybridization groups VII, 
TABLE 2. Herbicola-Agglomerans group strains

\begin{tabular}{|c|c|c|c|c|c|}
\hline $\begin{array}{c}\text { DNA } \\
\text { related- } \\
\text { ness } \\
\text { group }\end{array}$ & $\begin{array}{l}\text { Bio- } \\
\text { group }^{b}\end{array}$ & Strain designation ${ }^{c}$ & Name(s) as received & Source & Sent by: ${ }^{d}$ \\
\hline I & 1 & $2780-70$ & Enterobacter agglomerans & Human, foot wound & Washington SHD \\
\hline I & 1 & $5967-70$ & Enterobacter agglomerans & $\begin{array}{l}\text { Human, finger } \\
\text { wound }\end{array}$ & Montana SHD \\
\hline II & 1 & $238-70$ & Enterobacter agglomerans & Human, blood & New York SHD \\
\hline II & G1 & $1778-70$ & Enterobacter agglomerans & Human, blood & Oklahoma SHD \\
\hline II & 2 & $2548-70$ & Enterobacter agglomerans & Human, urine & Illinois SHD \\
\hline II & 2 & $2553-70$ & Enterobacter agglomerans & Human, leg wound & Hawaii SHD \\
\hline II & 2 & $3123-70$ & Enterobacter agglomerans & Human, stool & New Jersey SHD \\
\hline II & 1 & 198-71 & Enterobacter agglomerans & Human, urine & Montana SHD \\
\hline II & 1 & $217-71$ & Enterobacter agglomerans & Human, skin & U. Va. Hosp. \\
\hline II & 2 & $\begin{array}{l}258-71(=\text { ATCC } \\
29000)\end{array}$ & Enterobacter agglomerans & Human & Arizona SHD \\
\hline II & 1 & $303-71$ & Enterobacter agglomerans & Human, sputum & Virginia SHD \\
\hline II & $\mathrm{ND}^{e}$ & 876 & Enterobacter agglomerans & Human & Brenner \\
\hline III & 1 & $56-71$ & Enterobacter agglomerans & Human, blood & VA Hosp., Pittsburgh \\
\hline III & 2 & $226-71$ & Enterobacter agglomerans & Human, leg wound & Connecticut SHD \\
\hline III & 1 & $247-71$ & Enterobacter agglomerans & Human, blood & Florida SHD \\
\hline III & 2 & $1429-71$ & Enterobacter agglomerans & Intravenous fluid cap & Neblett \\
\hline III & 1 & $\begin{array}{l}\text { ATCC } 14589 \\
(=\text { ICPB EH118) }\end{array}$ & Erwinia herbicola & & Colwell \\
\hline IV & 3 & $2671-70$ & Enterobacter agglomerans & Human, urine & New Hampshire SHD \\
\hline IV & 6 & $3638-70$ & Enterobacter agglomerans & Human, cyst & Ft. McPherson, Ga. \\
\hline IV & 6 & $5795-70$ & Enterobacter agglomerans & Human, urine & PHS Hosp., Norfolk \\
\hline IV & 3 & $6148-70$ & Enterobacter agglomerans & Human, spinal fluid & Hawaii SHD \\
\hline IV & 3 & $\begin{array}{l}\text { 1741-71 ( } \\
27998)\end{array}$ & Enterobacter agglomerans & Human, trachea & Connecticut SHD \\
\hline $\mathrm{V}$ & 6 & $2928-68$ & Enterobacter agglomerans & Human, sputum & Wisconsin SHD \\
\hline $\mathrm{V}$ & 6 & $2525-70$ & Enterobacter agglomerans & & Quebec, Canada \\
\hline V & 3 & $4155-70$ & Enterobacter agglomerans & Human, nose & Connecticut SHD \\
\hline V & 2 & $4787-70$ & Enterobacter agglomerans & Human, throat & Pennsylvania SHD \\
\hline V & 3 & $3482-71$ & Enterobacter agglomerans & Human, urethra & Montana SHD \\
\hline V & 3 & $3518-71$ & Enterobacter agglomerans & Human, throat & $\begin{array}{l}\text { U.S. Army, } \\
\text { California }\end{array}$ \\
\hline $\mathrm{V}$ & G1 & $3527-71$ & Enterobacter agglomerans & Human, blood & $\begin{array}{l}\text { Institut Pasteur, } \\
\text { Paris, France }\end{array}$ \\
\hline V & 4 & $3737-71$ & Enterobacter agglomerans & Human, sputum & NIH \\
\hline VI & 4 & $3768-69$ & Enterobacter agglomerans & Environment & UNC \\
\hline VI & 4 & $5448-69$ & Enterobacter agglomerans & Human, blood & VA Hosp., Bronx \\
\hline VI & 4 & $6070-69$ & Enterobacter agglomerans & Human, wound & Georgia SHD \\
\hline VI & 4 & $5748-70$ & Enterobacter agglomerans & $\begin{array}{l}\text { Human, finger } \\
\text { wound }\end{array}$ & California SHD \\
\hline VI & 7 & XU104 & $\begin{array}{l}\text { "Xanthomonas uredovorus," Erwinia } \\
\text { uredovora }\end{array}$ & & Starr \\
\hline VI & 4 & EA181 & $\begin{array}{l}\text { Erwinia ananas, Erwinia herbicola } \\
\text { subsp. ananas }\end{array}$ & & Starr \\
\hline VI & 4 & B3526 & Enterobacter agglomerans & Human, blood & Weaver \\
\hline VI & 4 & $\begin{array}{l}\text { ATCC } 11530 \\
\quad(=\text { ICPB EA197) }\end{array}$ & $\begin{array}{l}\text { Erwinia ananas, Erwinia herbicola } \\
\text { subsp. ananas }\end{array}$ & & Colwell \\
\hline VI & 4 & $\begin{array}{l}\text { ATCC } 14536 \\
\quad(=\text { ICPB EH120) }\end{array}$ & $\begin{array}{l}\text { Erwinia ananas, Erwinia herbicola } \\
\text { subsp. ananas }\end{array}$ & & Colwell \\
\hline VI & 7 & $\begin{array}{l}\text { ATCC } 19321 \\
\quad(=\text { ICPB XU102) }\end{array}$ & Erwinia uredovora & & Colwell \\
\hline VI & 4 & $\begin{array}{l}\text { ATCC } 23822 \\
\quad(=\text { ICPB EH119) }\end{array}$ & $\begin{array}{l}\text { Erwinia ananas, Erwinia herbicola } \\
\text { subsp. ananas }\end{array}$ & Banana & Colwell \\
\hline VII & 5 & $166-70$ & Enterobacter agglomerans & Human, hand wound & NYCHD \\
\hline VII & 5 & $1469-70$ & Enterobacter agglomerans & Human, stool & Missouri SHD \\
\hline VII & 5 & $3970-70$ & Enterobacter agglomerans & Human, stool & North Carolina SHD \\
\hline VII & 5 & $4172-70$ & Enterobacter agglomerans & Human, stool & Alabama SHD \\
\hline VII & 5 & $5526-70$ & Enterobacter agglomerans & Human, eye & Louisiana SHD \\
\hline VII & 5 & $5656-70$ & Enterobacter agglomerans & Human, foot wound & Virginia SHD \\
\hline VII & 5 & $5685-71$ & Enterobacter agglomerans & Human & Massachusetts SHD \\
\hline VII & 5 & $6003-71$ & Enterobacter agglomerans & & New Jersey SHD \\
\hline VIII & 6 & $5422-69$ & Enterobacter agglomerans & Human, urine & Washington SHD \\
\hline VIII & 6 & $4707-72$ & Enterobacter agglomerans & $\begin{array}{l}\text { Human, pulmonary } \\
\text { infection }\end{array}$ & Mississippi SHD \\
\hline IX & G1 & $459-71 \mathrm{~A}$ & Enterobacter agglomerans & Human, skin & $\begin{array}{l}\text { U.S. Navy, } \\
\text { Portsmouth, Va. }\end{array}$ \\
\hline
\end{tabular}


TABLE 2-Continued

\begin{tabular}{|c|c|c|c|c|c|}
\hline $\begin{array}{l}\text { DNA } \\
\text { related- } \\
\text { ness } \\
\text { group }^{a}\end{array}$ & $\begin{array}{l}\text { Bio- } \\
\text { group }^{b}\end{array}$ & Strain designation ${ }^{c}$ & Name(s) as received & Source & Sent by: ${ }^{d}$ \\
\hline IX & G1 & 459-71B & Enterobacter agglomerans & Human, skin & $\begin{array}{l}\text { U.S. Navy, } \\
\text { Portsmouth, Va. }\end{array}$ \\
\hline IX & G1 & $2710-71$ & Enterobacter agglomerans & Mouse chow & NNMC \\
\hline IX & G4 & $3525-71$ & Enterobacter agglomerans & Human, nose & $\begin{array}{l}\text { Institut Pasteur, } \\
\text { Paris, France }\end{array}$ \\
\hline IX & G1 & $4388-71$ & Enterobacter agglomerans & Human, throat & NIH \\
\hline $\mathrm{X}$ & $\mathrm{G} 2$ & $1599-71$ & Enterobacter agglomerans & Chicken livers & New Hampshire SHD \\
\hline $\mathrm{X}$ & $\mathrm{G} 2$ & $1600-71$ & Enterobacter agglomerans & Chicken livers & New Hampshire SHD \\
\hline $\mathrm{XI}$ & G3 & $\begin{array}{l}1744-71 \\
3423)\end{array}$ & Enterobacter agglomerans & Human, leg wound & Connecticut SHD \\
\hline XI & G3 & $2709-71$ & Enterobacter agglomerans & Mouse chow & NIH \\
\hline XI & G3 & $2711-71$ & Enterobacter agglomerans & Human, urine & California SHD \\
\hline XI & G3 & $4519-71$ & Enterobacter agglomerans & Human, skin & Colorado SHD \\
\hline XI & G3 & $5378-71$ & Enterobacter agglomerans & Human, vagina & Missouri SHD \\
\hline XI & G3 & $\begin{array}{l}2674-72(=\mathrm{ATCC} \\
27984)\end{array}$ & Enterobacter agglomerans & Human, urine & Ft. McPherson, Ga. \\
\hline XII & G3 & $185-71$ & Enterobacter agglomerans & Human, blood & Pennsylvania SHD \\
\hline XII & G4 & $219-71$ & Enterobacter agglomerans & Intravenous fluid & U. Va. Hosp. \\
\hline XII & G4 & $299-71$ & Enterobacter agglomerans & Human, blood & New Jersey SHD \\
\hline XII & G4 & $509-71$ & Enterobacter agglomerans & Intravenous fluid & Michigan SHD \\
\hline XII & G3 & $934-71$ & Enterobacter agglomerans & Human, blood & Colorado SHD \\
\hline XII & G4 & $1083-71$ & Enterobacter agglomerans & Intravenous fluid & Neblett \\
\hline XII & G4 & $1309-71$ & Enterobacter agglomerans & Human, blood & Neblett \\
\hline XII & G4 & $1348-71$ & Enterobacter agglomerans & Human, blood & Colorado SHD \\
\hline XII & G4 & $1426-71$ & Enterobacter agglomerans & Intravenous fluid cap & Neblett \\
\hline XII & G4 & $3621-71$ & Enterobacter agglomerans & Human, hand wound & Florida SHD \\
\hline XII & G3 & $4176-71$ & Enterobacter agglomerans & Human, sputum & Colorado SHD \\
\hline XII & G4 & $4610-71$ & Enterobacter agglomerans & Human, sputum & VA Hosp., Durham \\
\hline XII & G4 & $5764-71$ & Enterobacter agglomerans & Human, urine & Georgia SHD \\
\hline XIII & 1 & $1645-71$ & Enterobacter agglomerans & & $\mathrm{CDC}$ \\
\hline XIII & 1 & $\begin{array}{l}2774-71(=\text { ICPB } \\
3424)\end{array}$ & Enterobacter agglomerans & Human, leg wound & Connecticut SHD \\
\hline XIII & 1 & $\underset{23374)}{\mathrm{EM} 101}(=\mathrm{ATCC}$ & $\begin{array}{l}\text { "Erwinia mangifera," Erwinia } \\
\text { herbicola subsp. herbicola }\end{array}$ & & Starr \\
\hline XIII & 1 & EL102 & $\begin{array}{l}\text { "Erwinia lathyri," Erwinia herbicola } \\
\text { subsp. herbicola }\end{array}$ & & $\begin{array}{l}\text { Starr } \\
\text { Starr }\end{array}$ \\
\hline XIII & 1 & $\underset{23375)}{\operatorname{EM} 102(=}$ ATCC & $\begin{array}{l}\text { Erwinia milletiae, Erwinia herbicola } \\
\text { subsp. herbicola }\end{array}$ & & Starr \\
\hline XIII & 1 & EH103 & Erwinia herbicola subsp. herbicola & & \\
\hline XIII & 1 & EL107 & $\begin{array}{l}\text { "Erwinia lathyri," Erwinia herbicola } \\
\text { subsp. herbicola }\end{array}$ & & Starr \\
\hline XIII & 1 & XТ109 & $\begin{array}{l}\text { "Xanthomonas trifolii," Erwinia } \\
\text { herbicola subsp. herbicola }\end{array}$ & & Starr \\
\hline XIII & 1 & EM114 & $\begin{array}{l}\text { Erwinia milletiae, Erwinia herbicola } \\
\text { subsp. herbicola }\end{array}$ & & Starr \\
\hline XIII & 1 & $\begin{array}{l}\text { ATCC } 12287 \\
\quad(=\text { ICPB EH117) }\end{array}$ & $\begin{array}{l}\text { "Xanthomonas trifolii," Erwinia } \\
\text { herbicola subsp. herbicola }\end{array}$ & & Colwell \\
\hline XIII & 1 & $\begin{array}{l}\text { ATCC } 23374 \\
(=\text { ICPB EM101) }\end{array}$ & $\begin{array}{l}\text { "Erwinia mangiferae," Erwinia } \\
\text { herbicola subsp. herbicola }\end{array}$ & & Colwell \\
\hline XIII & 1 & $\begin{array}{l}\text { ATCC } 23375 \\
(=\text { ICPB EM102) }\end{array}$ & $\begin{array}{l}\text { Erwinia milletiae, Erwinia herbicola } \\
\text { subsp. herbicola }\end{array}$ & & Colwell \\
\hline $\mathbf{U}$ & 7 & $5257-64$ & Enterobacter agglomerans & Human, urine & PHS Hosp. Norfolk \\
\hline $\mathrm{U}$ & 6 & $2116-68$ & Enterobacter agglomerans & Human, eye & South Carolina SHD \\
\hline $\mathrm{U}$ & 6 & $4132-68$ & Enterobacter agglomerans & Hospital environment & Yale University \\
\hline $\mathbf{U}$ & 6 & $26-69$ & Enterobacter agglomerans & Human, blood & California SHD \\
\hline $\mathrm{U}$ & 4 & $3618-69$ & Enterobacter agglomerans & Human, leg wound & California SHD \\
\hline $\mathbf{U}$ & 4 & $4953-69$ & Enterobacter agglomerans & Sheep & Mayo Clinic \\
\hline $\mathrm{U}$ & 1 & $831-70$ & Enterobacter agglomerans & $\begin{array}{l}\text { Human, finger } \\
\text { wound }\end{array}$ & New Hampshire SHD \\
\hline $\mathrm{U}$ & 3 & 893-70 & Enterobacter agglomerans & Human & Connecticut SHD \\
\hline $\mathrm{U}$ & 2 & $6012-70$ & Enterobacter agglomerans & Human & Georgia SHD \\
\hline $\mathrm{U}$ & 5 & $184-71$ & Enterobacter agglomerans & Human, urine & Pennsylvania SHD \\
\hline $\mathrm{U}$ & 2 & $314-71$ & Enterobacter agglomerans & Human, urine & Colorado SHD \\
\hline $\mathrm{U}$ & G1 & $1376-71$ & Enterobacter agglomerans & Human, blood & New Jersey SHD \\
\hline $\mathrm{U}$ & G2 & $1379-71$ & $\begin{array}{l}\text { Enterobacter agglomerans (actually } \\
\text { Erwinia cypripedii) }\end{array}$ & Intravenous fluid & Louisiana SHD \\
\hline $\mathrm{U}$ & G2 & $2499-71$ & Enterobacter agglomerans & Human & Pennsylvania SHD \\
\hline
\end{tabular}


TABLE 2-Continued

\begin{tabular}{|c|c|c|c|c|c|}
\hline $\begin{array}{c}\text { DNA } \\
\text { related- } \\
\text { ness } \\
\text { group }^{a}\end{array}$ & $\begin{array}{c}\text { Bio- } \\
\text { group }^{b}\end{array}$ & Strain designation ${ }^{\prime}$ & Name(s) as received & Source & Sent by: ${ }^{d}$ \\
\hline $\mathrm{U}$ & 2 & $2750-71$ & Enterobacter agglomerans & Human, vagina & Illinois SHD \\
\hline $\mathrm{U}$ & 1 & $2862-71$ & Enterobacter agglomerans & Mouse chow & $\mathrm{NIH}$ \\
\hline $\mathrm{U}$ & G4 & $4524-71$ & Enterobacter agglomerans & Human, skin & Colorado SHD \\
\hline $\mathrm{U}$ & $\mathrm{G} 2$ & $4908-71$ & Enterobacter agglomerans & & Hawaii SHD \\
\hline $\mathrm{U}$ & 3 & $4990-71$ & Enterobacter agglomerans & Rabbit, stool & NIH \\
\hline $\mathrm{U}$ & 7 & $5379-71$ & Enterobacter agglomerans & Human, spinal fluid & Illinois SHD \\
\hline $\mathbf{U}$ & G1 & $5380-71$ & Enterobacter agglomerans & $\begin{array}{l}\text { Human, abdominal } \\
\text { fluid }\end{array}$ & Montana SHD \\
\hline $\mathbf{U}$ & 7 & ATCC 23637 & Erwinia uredovora & Uredia of cereal rust & Colwell \\
\hline $\mathrm{U}$ & 1 & $\underset{23372)}{\mathrm{EC} 11(=\mathrm{ATCC}}$ & $\begin{array}{l}\text { "Erwinia cassavae," Erwinia herbicola } \\
\text { subsp. herbicola }\end{array}$ & & Starr \\
\hline $\mathrm{U}$ & 1 & $\begin{array}{l}\text { ATCC } 23372 \\
\quad(=\text { ICPB EC11) }\end{array}$ & $\begin{array}{l}\text { "Erwinia cassavae," Erwinia herbicola } \\
\text { subsp. herbicola }\end{array}$ & & Colwell \\
\hline $\mathrm{U}$ & G3 & $486-51$ & Enterobacter agglomerans & & Brenner \\
\hline $\mathrm{U}$ & 1 & ICPB 3163 & Erwinia herbicola & & Starr \\
\hline $\mathrm{U}$ & ND & NIH 457 & Enterobacter agglomerans & & Brenner \\
\hline $\mathrm{U}$ & 1 & EM110 & "Erwinia maydis" & & Starr \\
\hline $\mathrm{U}$ & 2 & ICPB 2553 & Erwinia herbicola & & Starr \\
\hline $\mathrm{U}$ & 4 & EA101 & Erwinia ananas & & Starr \\
\hline $\mathrm{U}$ & 7 & B3295 & Enterobacter agglomerans & Human, urine & Weaver \\
\hline $\mathrm{U}$ & 6 & B8006 & Enterobacter agglomerans & Human, urine & Weaver \\
\hline $\mathrm{U}$ & 6 & $\begin{array}{l}\text { ATCC } 14537 \\
\quad(=\text { ICPB EH116) }\end{array}$ & Erwinia herbicola & & Colwell \\
\hline $\mathrm{U}$ & 3 & $\begin{array}{l}\mathrm{SS} 11(=\mathrm{ATCC} \\
8199)\end{array}$ & Erwinia stewartii & & Starr \\
\hline
\end{tabular}

${ }^{a}$ A total of 13 DNA relatedness groups were identified in this study (see text). Strains that did not fit into any of these DNA groups are designated $U$.

${ }^{b}$ Biogroups of Ewing and Fife (14). G, Aerogenic.

${ }^{c}$ Strain designations with a hyphen (e.g., 2780-70) or beginning with a single letter (e.g., B3526) are Centers for Disease Control strains. Designations beginning with two letters (e.g., XU104) or with ICPB were obtained directly from the International Collection of Phytopathogenic Bacteria.

${ }^{d}$ SHD, State Health Department; U. Va. Hosp., University of Virginia Hospital, Charlottesville; Brenner, V. Brenner, National Institutes of Health, Bethesda, Md.; VA Hosp., Pittsburgh, Veterans Administration Hospital, Pittsburgh, Pa.; Neblett, T. R. Neblett, University of Michigan, Ann Arbor; Colwell, R. R. Colwell, University of Maryland, College Park; PHS Hosp., Norfolk, Public Health Service Hospital, Norfolk, Va.; NIH, National Institutes of Health, Bethesda, Md.; UNC, University of North Carolina, Chapel Hill; VA Hosp., Bronx, Veterans Administration Hospital, Bronx, N.Y.; Starr, M. P. Starr, International Collection of Phytopathogenic Bacteria; Weaver, R. E. Weaver, Centers for Disease Control, Atlanta, Ga.; NYCHD, New York City Health Department; NNMC, National Naval Medical Center, Bethesda, Md.; VA Hosp., Durham, Veterans Administration Hospital, Durham, N.C.; CDC, Centers for Disease Control, Atlanta, Ga.

${ }^{e} \mathrm{ND}$, Not determined.

IX, XI, and XII; hybridization group X was 20 to $30 \%$ related to the former hybridization groups and 30 to $39 \%$ related to the latter hybridization groups.

The reference strain from each hybridization group was tested against 25 to 30 strains which represented all of the recognized genera in the Enterobacteriaceae as of 1976. The levels of relatedness were between 4 and $56 \%$ (Table 4 ). The highest levels of relatedness were observed between Erwinia cypripedii and hybridization groups II through VI (41 to 47\%) and between Enterobacter species and hybridization group VII.

Strains isolated from humans were present in all 13 hybridization groups. A total of 20 strains isolated from humans or associated with human infections and 2 animal isolates did not fit into any of the hybridization groups. Strains isolated from plants (or presumed to have been isolated from plants by virtue of their Erwinia species names) were found in hybridization groups III, VI, and XIII, and nine such strains were ungroupable (Table 2).

The DNA hybridization groups did not correlate with the biogrouping scheme of Ewing and Fife (14). Seven hybridization groups contained strains from two or more biogroups, and strains from all biogroups except 5, 7, and G2 were found in more than one hybridization group. Biogroup 5 strains were found only in hybridization group VII, and biogroup G2 strains were found only in hybridization group 10 (only two strains). Strains belonging to all 11 biogroups were found among the strains that were ungroupable by DNA relatedness. Plant isolates were mainly found in biogroups 1,4 , and 7 , although single strains that were ungroupable by DNA relatedness were present in biogroups 2, 3, and 6.

Strains from aerogenic and anaerogenic biogroups were rarely in the same hybridization group. Hybridization groups IX through XII contained only aerogenic strains. A single aerogenic strain was included among the anaerogenic strains contained in hybridization groups II and V. The other hybridization groups contained only anaerogenic strains (Table 2). The aerogenic strain in hybridization group II (strain 1778-80) was only $69 \%$ related to the group II reference strain, and the aerogenic strain in hybridization group V (strain $3527-71$ ) was $73 \%$ related to the group V reference strain; the related sequences showed $6 \%$ divergence $\left(6^{\circ} \mathrm{C}\right.$ lower thermal stability than that observed for the homologous DNA from the reference strain). The other strains in hybridization group $\mathrm{V}$ were 83 to $100 \%$ related to 
TABLE 3. Levels of relatedness of the Herbicola-Agglomerans group strains in the 13 DNA hybridization groups

\begin{tabular}{|c|c|c|c|c|c|c|c|c|c|}
\hline $\begin{array}{l}\text { DNA } \\
\text { hybrid- } \\
\text { ization } \\
\text { group }\end{array}$ & Strain & $\begin{array}{c}\text { Relative } \\
\text { binding } \\
\text { ratio at } \\
60^{\circ} \mathrm{C}^{a}\end{array}$ & $\begin{array}{l}\text { Divergence } \\
\qquad(\%)^{b}\end{array}$ & $\begin{array}{c}\text { Relative } \\
\text { binding } \\
\text { ratio at } \\
75^{\circ} \mathrm{C}^{a}\end{array}$ & $\begin{array}{l}\text { DNA } \\
\text { hybrid- } \\
\text { ization } \\
\text { group }\end{array}$ & Strain & $\begin{array}{c}\text { Relative } \\
\text { binding } \\
\text { ratio at } \\
60^{\circ} \mathrm{C}^{a} \\
\end{array}$ & $\begin{array}{l}\text { Divergence } \\
(\%)^{b}\end{array}$ & $\begin{array}{c}\text { Relative } \\
\text { binding } \\
\text { ratio at } \\
75^{\circ} \mathrm{C}^{a} \\
\end{array}$ \\
\hline \multirow[t]{2}{*}{1} & $2780-70^{c}$ & 100 & 0.0 & 100 & & $3970-70$ & 90 & 1.0 & 84 \\
\hline & $5967-70$ & $93(93)^{d}$ & $0.0(0.0)$ & $94(94)$ & & $166-70$ & 88 & 2.0 & 79 \\
\hline \multirow[t]{10}{*}{ II } & $3123-70^{c}$ & 100 & 0.0 & 100 & & $5656-70$ & 87 & 1.5 & 82 \\
\hline & $2548-70$ & 100 & 0.5 & 96 & & $1469-70$ & 86 & 1.5 & 82 \\
\hline & $217-71$ & 96 & 0.0 & 96 & & $5685-71$ & $84(88)$ & $1.5(1.5)$ & $81(82)$ \\
\hline & $2553-70$ & 88 & 1.5 & 84 & VIII & $5422-69^{c}$ & 100 & 0.0 & 100 \\
\hline & 876 & 88 & 2.5 & 84 & & $4707-62$ & $97(97)$ & $0.5(0.5)$ & $91(91)$ \\
\hline & $198-71$ & 83 & 2.0 & 72 & IX & $4388-71^{c}$ & 100 & 0.0 & 100 \\
\hline & $258-71$ & 82 & 3.5 & 78 & & $3525-71$ & 100 & 0.0 & 100 \\
\hline & $238-70$ & 80 & 2.5 & 76 & & $2710-71$ & 91 & 1.5 & 83 \\
\hline & $303-71$ & 80 & 2.5 & 76 & & $459-71 \mathrm{~A}$ & 88 & 2.5 & 80 \\
\hline & $1778-70$ & $69(85)$ & $2.5(2.0)$ & $66(80)$ & & 459-71B & $85(91)$ & $3.0(2.0)$ & $74(84)$ \\
\hline \multirow[t]{5}{*}{ III } & $1429-71^{c}$ & 100 & 0.0 & 100 & $X$ & $1600-71^{c}$ & 100 & 0.0 & 100 \\
\hline & $226-71$ & 94 & 0.5 & 90 & & $1599-71$ & $100(100)$ & $0.0(0.0)$ & $97(97)$ \\
\hline & $56-71$ & 92 & 1.0 & 86 & XI & $5378-71^{c}$ & 100 & 0.0 & 100 \\
\hline & $247-71$ & 90 & 0.5 & 84 & & $2711-71$ & 75 & 5.5 & 57 \\
\hline & ATCC 14589 & $89(91)$ & $1.0(1.0)$ & $82(86)$ & & $4519-71$ & 74 & 5.5 & 63 \\
\hline \multirow[t]{5}{*}{ IV } & $1741-71^{c}$ & 100 & 0.0 & 100 & & $2709-71$ & 74 & 5.5 & 61 \\
\hline & $6148-70$ & 94 & 1.0 & 89 & & $2674-72$ & 73 & 6.0 & 59 \\
\hline & $5795-70$ & 91 & 0.0 & 92 & & $1744-71$ & $71(73)$ & $6.0(5.5)$ & $62(60)$ \\
\hline & $2671-70$ & 88 & 1.0 & 87 & XII & $219-71^{c}$ & 100 & 0.0 & 100 \\
\hline & $3638-70$ & $87(90)$ & $1.0(1.0)$ & $88(89)$ & & $1083-71$ & 100 & 0.0 & 99 \\
\hline \multirow[t]{8}{*}{ V } & $3482-71^{c}$ & 100 & 0.0 & 100 & & $1309-71$ & 99 & 0.0 & 98 \\
\hline & $2928-68$ & 100 & 0.0 & 100 & & $5764-71$ & 92 & 0.5 & 93 \\
\hline & $4787-70$ & 99 & 0.0 & 100 & & $4610-71$ & 90 & 0.5 & 92 \\
\hline & $3518-71$ & 99 & 0.0 & 100 & & $299-71$ & 88 & 1.5 & 84 \\
\hline & $3737-71$ & 99 & 0.0 & 98 & & $3621-71$ & 86 & 2.0 & 86 \\
\hline & $2525-70$ & 99 & 0.0 & 97 & & $1426-71$ & 84 & 6.0 & 70 \\
\hline & $4155-70$ & 84 & 0.0 & 84 & & $185-71$ & 83 & 6.0 & 66 \\
\hline & $3527-71$ & $73(93)$ & $6.0(1.0)$ & $67(92)$ & & $1348-71$ & 79 & 6.5 & 78 \\
\hline \multirow[t]{8}{*}{$\mathrm{VI}^{e}$} & $6070-69^{c}$ & 100 & 0.0 & 100 & & $509-71$ & 79 & 1.0 & 65 \\
\hline & B3526 & 90 & 0.0 & 90 & & 934-71 & 79 & 6.0 & 68 \\
\hline & $3768-69$ & 90 & 0.0 & 84 & & $4176-71$ & $75(86)$ & $6.0(3.0)$ & $66(82)$ \\
\hline & $5748-70$ & 89 & 0.0 & 84 & XIII & $1645-71^{c}$ & 100 & 0.0 & 100 \\
\hline & ATCC 19321 & 88 & 1.0 & 89 & & EM102 & 97 & 0.0 & 94 \\
\hline & XU104 & 85 & 0.5 & 85 & & ХT109 & 96 & 0.5 & 91 \\
\hline & $5448-69$ & 85 & 0.5 & 79 & & $2774-71$ & 96 & 1.0 & 91 \\
\hline & ATCC 11530 & $83(87)$ & $0.0(0.5)$ & $90(85)$ & & EL107 & 95 & 1.0 & 93 \\
\hline \multirow[t]{5}{*}{$\mathrm{VI}^{e}$} & ATCC $19321^{c}$ & 100 & 0.0 & & & EM114 & 93 & 1.0 & 94 \\
\hline & XU104 & 98 & 0.5 & & & ATCC 23375 & 92 & 1.0 & 92 \\
\hline & EA181 & 84 & 1.0 & & & EL102 & 92 & 2.0 & 89 \\
\hline & ATCC 23822 & 81 & 0.5 & & & EH103 & 88 & 1.0 & 90 \\
\hline & ATCC 14536 & $75(85)$ & $0.0(0.5)$ & & & ATCC 12287 & 87 & 0.0 & 83 \\
\hline \multirow[t]{3}{*}{ VII } & $6003-71^{c}$ & 100 & 0.0 & 100 & & EM101 & 80 & 6.0 & 69 \\
\hline & $4172-70$ & 91 & 1.5 & 83 & & ATCC 23374 & $75(90)$ & $6.5(2.0)$ & $68(87)$ \\
\hline & $5526-70$ & 91 & 2.0 & 84 & & & & & \\
\hline
\end{tabular}

${ }^{a}$ Relative binding ratios were calculated by using the following expression: [(percentage of heterologous DNA bound to hydroxyapatite)/ (percentage of homologous DNA bound to hydroxyapatite)] $\times 100$.

${ }^{b}$ Divergence was the decrease in the thermal stability of the heterologous DNA duplexes compared with the thermal stability of the homologous DNA duplexes. Divergence values were calculated on the assumption that each $1{ }^{\circ} \mathrm{C}$ decrease in thermal stability was due to approximately $1 \%$ unpaired bases in double-stranded DNA.

${ }^{c}$ Source of labeled DNA.

${ }^{d}$ The numbers in parentheses are averages. For each group the homologous DNA reaction was arbitrarily defined as $100 \%$. Therefore, the values for the homologous DNA reactions (labeled and unlabeled DNAs from the same strain) were excluded when the averages were calculated.

${ }^{e}$ Two labeled DNAs were used for DNA hybridization group VI. All reactions were done two or more times.

the reference strain, and their related sequences showed no divergence (Table 3). Therefore, one might argue that the aerogenic and anaerogenic biogroups are totally separable at the species level.

In hybridization group XI all strains were 71 to $75 \%$ related to the reference strain. The related sequences of these strains showed 5.5 to $6.0 \%$ divergence, and the level of relatedness fell to between 57 and $63 \%$ in reactions done at the stringent incubation temperature $\left(75^{\circ} \mathrm{C}\right)$. It is possible that all strains except the reference strain are very highly related (Table 3 ). The only other "heterogeneous" hybridization group was group XII, in which the level of relatedness was between 75 and $100 \%$, divergence was as high as $6.5 \%$, and the level of relatedness at $75^{\circ} \mathrm{C}$ was between 65 and $99 \%$ (Table 3 ).

Table 5 shows the results of 33 biochemical tests that were 
TABLE 4. Levels of relatedness of the Herbicola-Agglomerans group DNA hybridization groups to each other and to other members of the Enterobacteriaceae

\begin{tabular}{|c|c|c|c|c|c|}
\hline \multirow{2}{*}{ Source of unlabeled DNA } & \multicolumn{5}{|c|}{ Relative binding ratio at $60^{\circ} \mathrm{C}$ with DNA hybridization group: ${ }^{a}$} \\
\hline & I & II & III & IV & V \\
\hline $\mathrm{HG} \mathrm{I}^{b}$ & 93 & $45(1)^{c}$ & $44(1)$ & $42(1)$ & $38(1)$ \\
\hline HG II & $49 \pm 1.8(10)^{e}$ & 85 & $56 \pm 1.4(2)$ & $53 \pm 4.9(2)$ & $53 \pm 4.9(2)$ \\
\hline HG III & $47 \pm 0.9(5)$ & $51 \pm 3.5(5)$ & 91 & $59 \pm 2.3(5)$ & $50 \pm 4.1(5)$ \\
\hline HG IV & $43 \pm 4.1(5)$ & $44 \pm 3.8(5)$ & $56(1)$ & 90 & $44 \pm 5.4(5)$ \\
\hline HG V & $44 \pm 2.0(8)$ & $50 \pm 1.4(8)$ & $56(1)$ & $50 \pm 2.4(8)$ & 93 \\
\hline HG VI & $36 \pm 2.9(11)$ & $38 \pm 3.4(11)$ & $47 \pm 2.8(2)$ & $46 \pm 2.1(2)$ & $51 \pm 0.7(2)$ \\
\hline HG VII & $36 \pm 2.0(8)$ & $30 \pm 1.2(8)$ & $37(1)$ & $34 \pm 2.1(2)$ & $33(1)$ \\
\hline HG VIII & $49 \pm 0.7(2)$ & $42 \pm 0.7(2)$ & $46 \pm 3.5(2)$ & $39 \pm 4.9(2)$ & $45 \pm 1.4(2)$ \\
\hline HG IX & $41 \pm 3.3(5)$ & $33 \pm 0.0(2)$ & $38 \pm 4.9(2)$ & $29 \pm 7.1(2)$ & $36 \pm 0.7(2)$ \\
\hline HG $\mathrm{X}$ & $31 \pm 2.8(2)$ & $24(1)$ & $30(1)$ & $23(1)$ & $24(1)$ \\
\hline HG XI & $35 \pm 2.4(6)$ & $33 \pm 1.6(6)$ & $39(1)$ & $30 \pm 5.1(3)$ & $34 \pm 6.1(3)$ \\
\hline HG XII & $31 \pm 2.9(13)$ & $27 \pm 2.1(13)$ & $30(1)$ & $25 \pm 2.1$ & $29 \pm 4.3(4)$ \\
\hline HG XIII & $42 \pm 1.8(12)$ & $48 \pm 1.9(4)$ & $50 \pm 0.6(3)$ & $46 \pm 5.2(3)$ & $64 \pm 2.0(3)$ \\
\hline Erwinia cypripedii & $-^{f}$ & 41 & 47 & 41 & 41 \\
\hline Erwinia salicis & - & 36 & - & - & - \\
\hline Erwinia amylovora & - & - & - & - & - \\
\hline $\begin{array}{l}\text { Escherichia, Shigella, Salmonella, Enterobacter, } \\
\text { Klebsiella, Citrobacter }{ }^{g}\end{array}$ & $25-35$ & $25-31$ & $25-35$ & $27-36$ & $.25-32$ \\
\hline $\begin{array}{l}\text { Erwinia, Serratia, Hafnia, Yersinia, } \\
\text { Edwardsiella, Morganella }{ }^{g}\end{array}$ & $17-25$ & $13-23$ & $16-24$ & $21-26$ & $19-25$ \\
\hline Providencia, Proteus ${ }^{g}$ & $7-9$ & $5-8$ & $8-10$ & $9-11$ & $6-9$ \\
\hline
\end{tabular}

${ }^{a}$ See Table 3, footnote $a$. Standard errors of the mean are given except for reactions among strains of the same hybridization group.

${ }^{b}$ HG, DNA hybridization group.

${ }^{c}$ The numbers in parentheses are the numbers of strains tested.

${ }^{d}$ ND, Not determined.

${ }^{e}$ Data are expressed as mean \pm standard error.

$f$, , Value in the range given for the genus.

${ }^{g}$ Labeled DNA from each DNA hybridization group was tested for its level of relatedness to 25 to 30 species of the genera indicated.

helpful in distinguishing among the hybridization groups. Only one API 50R test helped differentiate among hybridization groups. Several API 50R tests were not done in the conventional system; the tests which gave uniformly positive or negative results are shown in Table 5, footnote $a$. Groups I, VIII, and X, which contained only two strains each, were omitted, as were strains that did not fit into any hybridization group. Table 5 shows that it was difficult to distinguish biochemically among several of the hybridization groups, especially groups II through V. One simplistic approach is presented in Fig. 1. Groups VI, VII, IX, XI, XII, and XIII are separable from one another and from the other hybridization groups on the basis of gas production and several commonly used tests. Hybridization group IV is separable from groups II, III, and V by means of its negative malonate and rhamnose reactions. Groups II, III, and V are only partially separable on the basis of their reactions for cellobiose and acetate. The fermentation of dextrin in the API 50R system was helpful, as group V strains were positive and group II and III strains were negative in this test.

A total of 72 strains representing all 13 hybridization groups were compared by numerical taxonomy. There were two types of relationship between hybridization groups and phenotypic clustering behavior. All aerogenic strains (hybridization groups IX through XII) and the anaerogenic strains of hybridization groups I, VI, and VII formed distinct phenotypic clusters, whereas the strains of the other hybridization groups did not form homogeneous clusters. Strains that were not grouped by DNA hybridization did not cluster together.

Antimicrobial agent susceptibility tests were performed on 74 strains chosen from all of the hybridization groups, as well as from strains that could not be grouped by DNA hybridization. All of the strains tested were susceptible to chloramphenicol, polymyxin b, and cefamandole; all but one to three strains were susceptible to amikacin, cefoxitin, gentamicin, kanamycin, tetracycline, tobramycin, and trimethoprim-sulfamethoxazole. Resistance to other antimicrobial agents was as follows: ampicillin, 17 strains (plus 2 strains showing zone sizes intermediate between susceptibility and resistance); carbenicillin, 20 strains (plus 15 strains with intermediate zone sizes); cephalothin, 6 strains (plus 6 strains with intermediate zone sizes); nalidixic acid, 1 strain (plus 5 strains with intermediate zone sizes); nitrofurantoin, 23 strains (plus 4 strains with intermediate zone sizes); and penicillin $G, 57$ strains (plus 4 strains with intermediate zone sizes). Resistant strains were found in all hybridization groups except groups VII, X, and XI. The antimicrobial agent susceptibility patterns were not helpful in distinguishing among the hybridization groups.

\section{DISCUSSION}

The DNA relatedness studies on the Herbicola-Agglomerans group were completed during 1974 and were reported preliminarily in 1975 (G. R. Fanning, A. G. Steigerwalt, P. Klykken, E. Cadet, and D. J. Brenner, Abstr. Annu. Meet. Am. Soc. Microbiol. 1975, C52, p. 35).

The phenotypic characteristics described here were determined in 1976 and 1977. Although the hybridization groups that contained aerogenic strains and several of the groups containing anaerogenic strains could be identified phenotypically, four hybridization groups (groups II through V) were difficult, if not impossible, to define and separate biochemically. Analyzing the phenotypic data by numerical taxonom- 
TABLE 4-Continued

\begin{tabular}{|c|c|c|c|c|c|c|c|}
\hline \multicolumn{8}{|c|}{ Relative binding ratio at $60^{\circ} \mathrm{C}$ with DNA hybridization group: ${ }^{a}$} \\
\hline VI & VII & VIII & IX & $\mathrm{X}$ & XI & XII & XIII \\
\hline $36(1)$ & $32(1)$ & $41(1)$ & $35(1)$ & $27(1)$ & $20(1)$ & $29(1)$ & $\mathrm{ND}^{d}$ \\
\hline $45 \pm 4.9(2)$ & $34 \pm 0.7(2)$ & $44 \pm 2.1(2)$ & $33 \pm 2.8(2)$ & $26 \pm 1.4(2)$ & $21 \pm 0.0(2)$ & $32 \pm 1.4(2)$ & $49(1)$ \\
\hline $47 \pm 1.7(5)$ & $33 \pm 2.8(5)$ & $44 \pm 2.3(5)$ & $38 \pm 1.9(5)$ & $27 \pm 0.4(5)$ & $21 \pm 3.0(5)$ & $33 \pm 4.7(5)$ & $45(1)$ \\
\hline $43 \pm 2.3(5)$ & $27 \pm 5.6(5)$ & $39 \pm 3.2(5)$ & $34 \pm 1.9(5)$ & $24 \pm 0.4(5)$ & $19 \pm 3.3(5)$ & $28 \pm 4.0(5)$ & ND \\
\hline $51 \pm 2.0(8)$ & $30 \pm 3.8(8)$ & $44 \pm 1.4(8)$ & $36 \pm 3.5(8)$ & $27 \pm 2.6(8)$ & $22 \pm 2.3(8)$ & $30 \pm 3.6(8)$ & ND \\
\hline 87 & $27 \pm 1.4(2)$ & $38 \pm 1.4(2)$ & $32 \pm 2.1(11)$ & $24 \pm 1.6(11)$ & $17 \pm 4.1(8)$ & $28 \pm 5.7(8)$ & $43 \pm 3.4(4)$ \\
\hline $31 \pm 2.0(8)$ & 88 & $34 \pm 2.0(8)$ & $49 \pm 1.2(8)$ & $38 \pm 1.4(8)$ & $54 \pm 2.6(8)$ & $45 \pm 1.1(8)$ & ND \\
\hline $42 \pm 0.7(2)$ & $27 \pm 1.4(2)$ & 97 & $36 \pm 1.4(2)$ & $30 \pm 1.4(2)$ & $21 \pm 2.1(2)$ & $38 \pm 7.1(2)$ & ND \\
\hline $30 \pm 0.0(2)$ & $48 \pm 3.5$ & $35 \pm 6.4(2)$ & 91 & $36 \pm 0.7(2)$ & $39 \pm 2.8(2)$ & $49 \pm 2.8(2)$ & ND \\
\hline $26(1)$ & 39 (1) & $31(1)$ & $37(1)$ & 100 & $23(1)$ & $34(1)$ & ND \\
\hline $30 \pm 2.6(11)$ & $58 \pm 1.7$ & $32 \pm 0.6(2)$ & $48 \pm 1.5(6)$ & $37 \pm 2.6(6)$ & 73 & $44 \pm 1.5(6)$ & ND \\
\hline $31 \pm 4.1(4)$ & $44 \pm 3.6$ & $29 \pm 3.0(4)$ & $49 \pm 2.4(13)$ & $36 \pm 1.8(13)$ & $36 \pm 2.8$ & 86 & ND \\
\hline $48 \pm 5.9(3)$ & $28 \pm 0.6(3)$ & $40 \pm 1.5(3)$ & $34 \pm 1.0(4)$ & $26 \pm 0.8$ & $19 \pm 2.3(3)$ & $29 \pm 1.5(3)$ & 90 \\
\hline 42 & 27 & 31 & 30 & 26 & 20 & 28 & 39 \\
\hline - & - & - & - & - & - & - & - \\
\hline - & - & 41 & - & - & - & - & - \\
\hline $24-30$ & $39-56$ & $24-32$ & $37-49$ & $33-39$ & $31-45$ & $38-44$ & $23-29$ \\
\hline $17-23$ & $17-28$ & $17-23$ & $19-34$ & $20-29$ & $12-26$ & $15-28$ & $16-23$ \\
\hline $7-12$ & $8-11$ & $7-8$ & $8-13$ & $8-11$ & $4-8$ & $10-16$ & \\
\hline
\end{tabular}

ic techniques did not improve our ability to distinguish among these hybridization groups.

Diagnostic bacteriologists were still faced with the problem of identifying clinical isolates of this complex on the basis of a biochemical data base that clearly contained data from at least several different species, and there was concern that one or more of the newly defined species, such as Escherichia vulneris (4), in the family Enterobacteriaceae might be a synonym for one of the names on the Approved Lists. For these reasons we decided to present the data here, to point out the shortcomings of this study, and to try to define the scope of further studies.

Of the 124 strains of the Herbicola-Agglomerans group which we studied by DNA relatedness, 90 formed 13 distinct hybridization groups (average relatedness, $73 \%$ or more for each group). The remaining 34 strains did not fit into any of these hybridization groups. The levels of relatedness among the 13 hybridization groups were 17 to $64 \%$. The levels of relatedness between the Herbicola-Agglomerans group strains and other Enterobacteriaceae strains were highest with some species of Erwinia and Enterobacter, followed by species of Escherichia, Shigella, Salmonella, Citrobacter, and Klebsiella. In no case was the level of relatedness between a hybridization group strain and another Enterobacteriaceae strain as high as the level of relatedness between that hybridization group and at least one other hybridization group in the Herbicola-Agglomerans complex. From these data we are confident that each hybridization group represents a single, unique species within the family Enterobacteriaceae.

A total of 26 plant isolates were studied. A single Erwinia herbicola strain was in hybridization group III. Hybridization group VI contained two strains of Erwinia uredovora and four strains of Erwinia ananas. Ten plant strains were in hybridization group XIII. These included strains received as "Erwinia lathyri," "Erwinia mangiferae," " $X$. trifolii," and Erwinia milletiae (all considered to be synonyms of Erwinia herbicola subsp. herbicola), as well as Erwinia herbicola subsp. herbicola. Nine plant isolates did not fit into any of the 13 hybridization groups. These strains were received as Erwinia herbicola subsp. herbicola, Erwinia herbicola subsp. ananas, Erwinia uredovora, Erwinia stewartii, "Erwinia maydis," and "Erwinia cassavae." These results show that the present classification of members of the Herbicola-Agglomerans group as three species plus Erwinia herbicola subsp. ananas is inadequate.

The classification scheme of Ewing and Fife with all of the strains in Enterobacter agglomerans and subdivision of the strains into 11 biogroups (14) is also not supported by the present data since there are multiple species, since more than one biogroup is present in 7 of the 10 hybridization groups that contain more than two strains, and since strains from almost all biogroups are present in more than one hybridization group. However, aerogenic strains and anaerogenic strains were not found in the same hybridization group.

Previous studies showed that $70 \%$ or greater interrelatedness was characteristic of the strains within any species of the Enterobacteriaceae $(4,15,30)$. By this criterion there are 13 species among the grouped strains and 5 or more species among the 34 strains that do not fit into any of the groups (4 of these strains were labeled and were not related at the species level to any of the other ungroupable strains).

If we dismiss the ungroupable strains and hybridization group $\mathrm{X}$ (two strains isolated simultaneously from chicken livers), we are left with 12 species that contain two or more independently isolated strains. Seven of these hybridization groups are separable on the basis of phenotypic characteristics and, except for group VIII, form highly related clusters as determined by numerical taxonomic analysis (groups I, VI, VII, IX, XI, and XII). Groups II through V and XIII cannot be separated with certainty on the basis of biochemical tests, although phenylalanine deaminase, malonate, rhamnose, cellobiose, acetate, and dextrin reactions are helpful in distinguishing them.

Since 1980 , a species has standing in nomenclature only if it appears on the Approved Lists (28). Seven species names that represent members of the Herbicola-Agglomerans group 
TABLE 5. Differential reactions among DNA hybridization groups of the Herbicola-Agglomerans group

\begin{tabular}{|c|c|c|c|c|c|c|c|c|c|c|}
\hline \multirow[b]{2}{*}{ Test } & \multicolumn{10}{|c|}{ \% Of positive reactions in DNA hybridization group: ${ }^{a}$} \\
\hline & $\begin{array}{c}\text { II } \\
\text { (8 strains) }\end{array}$ & $\begin{array}{c}\text { III } \\
\text { (7 strains) }\end{array}$ & $\begin{array}{c}\text { IV } \\
\text { (5 strains) }\end{array}$ & $\begin{array}{c}\mathrm{V} \\
\text { (8 strains) }\end{array}$ & $\begin{array}{c}\text { VI } \\
\text { (4 strains) }\end{array}$ & $\begin{array}{c}\text { VII } \\
\text { (8 strains) }\end{array}$ & $\begin{array}{c}\text { IX } \\
\text { (3 strains) }\end{array}$ & $\begin{array}{c}\mathrm{XI} \\
\text { (6 strains) }\end{array}$ & $\begin{array}{c}\text { XII } \\
\text { (14 strains) }\end{array}$ & $\begin{array}{c}\text { XIII } \\
\text { (3 strains) }\end{array}$ \\
\hline Urea & 25 & 0 & 20 & 37 & 25 & 75 & 33 & 100 & 28 & 33 \\
\hline Indole & 0 & 14 & 0 & 12 & 100 & 100 & 0 & 100 & 7 & 33 \\
\hline Methyl red & 75 & 71 & 80 & 62 & 0 & 100 & 0 & 100 & 35 & 33 \\
\hline Voges-Proskauer & 50 & 28 & 40 & 50 & 100 & 0 & 100 & 0 & 78 & 100 \\
\hline Citrate (Simmons) & 100 & 87 & 100 & 87 & 75 & 25 & 100 & 16 & 100 & 66 \\
\hline Growth in $\mathrm{KCN}$ & 0 & 14 & 20 & 25 & 25 & 100 & 66 & 83 & 78 & 33 \\
\hline Motility & 75 & 100 & 80 & 75 & 100 & 100 & 100 & 100 & 7 & 100 \\
\hline Gelatin $\left(22^{\circ} \mathrm{C}\right)$ & 75 & 82 & 60 & 100 & 100 & 75 & 100 & 33 & 85 & 100 \\
\hline Phenylalanine deaminase & 0 & 33 & 20 & 25 & 0 & 0 & 33 & 0 & 14 & 100 \\
\hline Gas from D-glucose & 0 & 14 & 0 & 12 & 0 & 0 & 66 & 100 & 100 & 0 \\
\hline Lactose & 37 & 42 & 20 & 37 & 100 & 100 & 33 & 100 & 100 & 100 \\
\hline Sucrose & 87 & 57 & 100 & 75 & 100 & 0 & 66 & 66 & 100 & 100 \\
\hline Dulcitol & 0 & 0 & 0 & 25 & 0 & 0 & 66 & 66 & 21 & 0 \\
\hline Salicin & 62 & 100 & 20 & 50 & 75 & 100 & 100 & 100 & 100 & 100 \\
\hline Adonitol & 0 & 0 & 0 & 12 & 0 & 0 & 0 & 83 & 0 & 33 \\
\hline i-Inositol & 62 & 42 & 20 & 50 & 100 & 0 & 33 & 16 & 0 & 0 \\
\hline D-Sorbitol & 0 & 14 & 0 & 12 & 100 & 0 & 100 & 16 & 93 & 0 \\
\hline L-Arabinose & 100 & 100 & 100 & 87 & 100 & 100 & 100 & 100 & 100 & 100 \\
\hline Raffinose & 0 & 0 & 0 & 25 & 100 & 12 & 66 & 83 & 42 & 0 \\
\hline L-Rhamnose & 100 & 100 & 20 & 75 & 100 & 100 & 100 & 100 & 100 & 100 \\
\hline Malonate & 62 & 71 & 0 & 87 & 75 & 100 & 66 & 83 & 93 & 66 \\
\hline Mucate & 62 & 28 & 20 & 25 & 25 & 0 & 100 & 0 & 100 & 33 \\
\hline Tartrate (Jordan) & 0 & 28 & 0 & 0 & 0 & 0 & 0 & 16 & 7 & 0 \\
\hline Acetate & 12 & 85 & 40 & 37 & 100 & 62 & 66 & 100 & 93 & 33 \\
\hline D-Xylose & 100 & 85 & 80 & 100 & 100 & 100 & 100 & 100 & 93 & 100 \\
\hline Trehalose & 100 & 100 & 100 & 100 & 0 & 87 & 100 & 100 & 93 & 0 \\
\hline Cellobiose & 25 & 71 & 80 & 87 & 100 & 100 & 100 & 100 & 100 & 66 \\
\hline Glycerol & 37 & 14 & 0 & 50 & 100 & 75 & 100 & 66 & 64 & 33 \\
\hline$\alpha$-Methyl glucoside & 0 & 0 & 0 & 0 & 25 & 0 & 0 & 0 & 64 & 0 \\
\hline Esculin & 100 & 100 & 60 & 87 & 50 & 100 & 100 & 100 & 100 & 100 \\
\hline Citrate (Christensen) & 100 & 100 & 100 & 100 & 0 & 62 & 100 & 16 & 93 & 0 \\
\hline $\mathrm{NO}_{3}{ }^{-} \rightarrow \mathrm{NO}_{2}^{-}$ & 100 & 100 & 60 & 25 & 0 & 100 & 100 & 100 & 78 & 100 \\
\hline Pigment (yellow) & 87 & 42 & 60 & 87 & 100 & 87 & 66 & 83 & 0 & 66 \\
\hline
\end{tabular}

${ }^{a}$ All results are for conventional biochemical tests performed on conventional media and incubated at $36 \pm 1{ }^{\circ} \mathrm{C}$ for $48 \mathrm{~h}$. All strains gave positive reactions in tests for growth on MacConkey agar and acid production from D-glucose, D-mannitol, D-arabitol, L-arabinose, (API 50R test), ribose (API 50R test), L-xylose (API 50R test), galactose (API 50R test), D-levulose (API 50R test), and D-mannose (API 50R test) and negative reactions in tests for gram staining, oxidase, $\mathrm{H}_{2} \mathrm{~S}$, pectate (API 50R test), lysine decarboxylase, arginine dihydrolase, ornithine decarboxylase, lipase (corn oil), deoxyribonuclease (API 50R test), and acid production from erythritol, methyl xyloside (API 50R test), Lsorbose (API 50R test), alpha-methyl-D-mannoside (API 50R test), $N$-acetylglucosamine (API 50R test), inulin (API 50R test), D-melizitose (API 50R test), amylose (API 50R test), and glycogen (API 50R test).

appear on the Approved Lists. Two of these, Erwinia stewartii (strain ATCC 8199 [= SS11]) and Erwinia uredovora (strain ATCC 19321) were used in this study. Erwinia stewartii ATCC 8199 was not grouped, and Erwinia uredovora ATCC 19321 was in hybridization group VI. The other type strains used were either not designated when the study was initiated, were poorly known, or, for Enterobacter agglomerans ATCC 27155, were inexplicably excluded. Species are defined in terms of their type strains. For example, if the type strain of Erwinia herbicola were in hybridization group $\mathrm{A}$ and 10 other strains called Erwinia herbicola were in hybridization group $\mathrm{B}$, hybridization group A would be Erwinia herbicola. Alternatively, if two type strains were in the same hybridization group, the first name to be published would have priority and the second name would lose standing in nomenclature. There are more than enough hybridization groups to accommodate all of the species names, but it will be necessary to examine all type strains before making any nomenclatural proposal.

Another problem in classification is the designation of one or more genus names for members of the Herbicola-Agglomerans group. The designated species are presently in three genera, Erwinia, Enterobacter, and Escherichia (Escherichia adecarboxylata). This problem can be approached logically only after the species problems have been answered.

The present study suffered from several problems in addition to the lack of type strains. We thought that the total number of strains chosen for study (124 strains) was more than adequate. Unfortunately, we were wrong. There were not enough plant strains, and Escherichia adecarboxylata strains were not included. The human strains were mainly from a nationwide outbreak of septicemia (24). The strains certainly showed genetic diversity, so much that 90 strains formed 13 hybridization groups and 34 other strains were ungroupable. This resulted in a small number of strains with which to define the biochemical characteristics of most hybridization groups. The lack of a sufficient number of strains does not account for our inability to separate each hybridization group on the basis of biochemical tests. It is possible that some, but not all, of the problem was technical.

Since we could not separate all hybridization groups with common biochemical tests, additional tests should be investigated. The possibilities include substrate utilization tests, specific enzyme substrate tests, and tests for differences in 


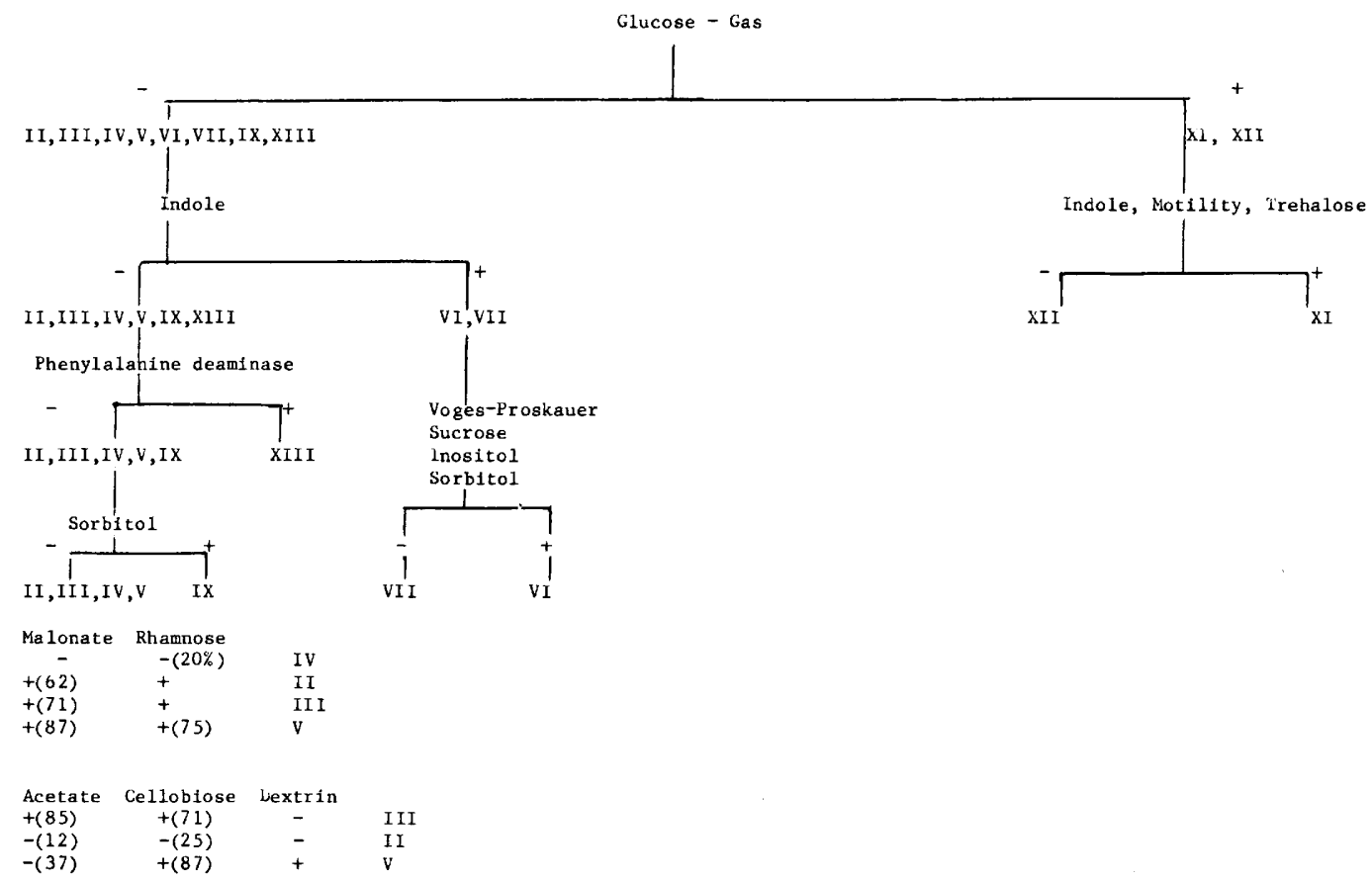

FIG. 1. Key for biochemical differentiation of the hybridization groups of Enterobacter agglomerans. The numbers in parentheses indicate the percentages of positive reactions after $48 \mathrm{~h}$ of incubation at $36 \pm 1{ }^{\circ} \mathrm{C}$; where there are no numbers in parentheses, $100 \%$ of the reactions gave the results shown.

growth temperature range. On a practical note, several of the biochemical tests that helped identify members of the Herbicola-Agglomerans group are not included in many of the commercially available identification systems. These tests include trehalose, cellobiose, malonate, and acetate.

\section{ACKNOWLEDGMENTS}

We are indebted to P. Klykken and E. Cadet for technical assistance in some of the DNA hybridization experiments.

\section{LITERATURE CITED}

1. Bauer, A. W., W. M. M. Kirby, J. C. Sherris, and M. Turck. 1966. Antibiotic sensitivity testing by a standardized disk method. Am. J. Clin. Pathol. 45:493-496.

2. Beijerinck, M. W. 1888. Cultur des Bacillus radicicola aus den Knollchen. Bot. Zeitung 46:740-750.

3. Bottone, E., and S. S. Schneierson. 1972. Erwinia species: an emerging human pathogen. Am. J. Clin. Pathol. 57:400-405.

4. Brenner, D. J., A. C. McWhorter, J. K. Leete Knudson, and A. G. Steigerwalt. 1982. Escherichia vulneris: a new species of Enterobacteriaceae associated with human wounds. J. Clin. Microbiol. 15:1133-1140.

5. Cooper-Smith, M. E., and A. von Graevenitz. 1978. Nonepidemic Erwinia herbicola (Enterobacter agglomerans) in blood cultures: bacteriological analysis of fifteen cases. Curr. Microbiol. 1:29-32.

6. Cruickshank, J. C. 1935. A study of the so-called Bacterium typhi flavum. J. Hyg. 35:354-371.

7. Dresel, E. G., and O. Stickl. 1928. Uber reversible Mutationsformen der Typhusbazillen beim Menschen. Dtsch. Med. Wochenschr. 54:517-519.

8. Dye, D. W. 1963. The taxonomic position of Xanthomonas uredovorus Pon et al. 1954. N. Z. J. Sci. 6:146-149.

9. Dye, D. W. 1963. The taxonomic position of Xanthomonas stewartii (Erw. Smith 1914) Dowson 1939. N. Z. J. Sci. 6:495506.

10. Dye, D. W. 1964. The taxonomic position of Xanthomonas trifolii (Huss, 1907) James, 1955. N. Z. J. Sci. 7:261-269.
11. Dye, D. W. 1966. A comparative study of some atypical "xanthomonads." N. Z. J. Sci. 9:843-854.

12. Dye, D. W. 1969. A taxonomic study of the genus Erwinia. III. The "Herbicola" group. N. Z. J. Sci. 12:223-236.

13. Ewing, W. H., and M. A. Fife. 1971. Enterobacter agglomerans, the Herbicola-Lathyri bacteria. Center for Disease Control, Atlanta, Ga.

14. Ewing, W. H., and M. A. Fife. 1972. Enterobacter agglomerans (Beijerinck) comb. nov. (the Herbicola-Lathyri bacteria). Int. J. Syst. Bacteriol. 22:4-11.

15. Farmer, J. J., III, G. R. Fanning, G. P. Huntley-Carter, B. Holmes, F. W. Hickman, C. Richard, and D. J. Brenner. 1981. Kluyvera, a new (redefined) genus in Enterobacteriaceae: identification of $K$. ascorbata sp. nov. and $K$. cryocrescens $\mathrm{sp}$. nov. in clinical specimens. J. Clin. Microbiol. 13:919-933.

16. Geere, I. W. 1977. Enterobacter agglomerans: the clinically important plant pathogen. Can. Med. Assoc. J. 116:517-519.

17. Gilardi, G. L., and E. W. Bottone. 1971. Erwinia and yellowpigmented Enterobacter isolates from human sources. Antonie von Leeuwenhoek J. Microbiol. Serol. 37:529-535.

18. Graham, D. C., and W. Hodgkiss. 1967. Identity of gram negative, yellow pigmented, fermentative bacteria isolated from plants and animals. J. Appl. Bacteriol. 30:175-189.

19. Graham, D. C., and C. E. Quinn. 1974. Identification of Agrobacterium gypsophilae strains NCPPB 179 and NCPPB 1948 as Erwinia herbicola. Int. J. Syst. Bacteriol. 24:238-241.

20. Hayward, A. C., and W. Hodgkiss. 1961. Taxonomic relationships of Xanthomonas uredovorus. J. Gen. Microbiol. 26:133140 .

21. Hickman, F. W., and J. J. Farmer III. 1978. Salmonella typhi: identification, antibiograms, serology, and bacteriophage typing. Am. J. Med. Technol. 44:1149-1159.

22. Leclerc, H. 1962. Etude biochimique d'Enterobacteriaceae pigmentées. Ann. Inst. Pasteur Paris 102:726-741.

23. Lelliott, R. A. 1974. Genus XII. Erwinia Winslow, Broadhurst, Buchanan, Krumwiede, Rogers and Smith 1920, 209, p. 332-339. In R. E. Buchanan and N. E. Gibbons (ed.), Bergey's manual of determinative bacteriology, 8th ed. The Williams \& Wilkins Co., Baltimore.

24. Maki, D. G., F. S. Rhame, D. C. Mackel, and J. V. Bennett. 
1976. Nationwide epidemic of septicemia caused by contaminated intravenous products. I. Epidemiologic and clinical features. Am. J. Med. 60:471-485.

25. Muraschi, T. F., M. Friend, and D. Bolles. 1965. Erwinia-like microorganisms isolated from animal and human hosts. Appl. Microbiol. 13:128-131.

26. Richard, C. 1974. Une nouvelle espéce d'entérobactérie rencontrée en bactériologie médicale Enterobacter agglomerans (exErwinia herbicola). Ann. Biol. Clin. (Paris) 32:341-346.

27. Schneierson, S. S., and E. J. Bottone. 1973. Erwinia infections in man. Crit. Rev. Clin. Lab. Sci. 4:341-355.

28. Skerman, V. B. D., V. McGowan, and P. H. A. Sneath (ed.). 1980. Approved lists of bacterial names. Int. J. Syst. Bacteriol. 30:225-420.

29. Sneath, P. H., and R. R. Sokal. 1973. Numerical taxonomy. W. H. Freeman and Co., San Francisco.

30. Steigerwalt, A. G., G. R. Fanning, M. A. Fife-Asbury, and D. J. Brenner. 1976. DNA relatedness among species of Enterobacter and Serratia. Can. J. Microbiol. 22:121-137.
31. Ursing, J. 1977. Characterization of Enterobacter agglomerans (Erwinia spp.) from clinical specimens. Acta Pathol. Microbiol. Scand. Sect. B 85:61-66.

32. von Graevenitz, A. 1970. Erwinia species isolates. Ann. N.Y. Acad. Sci. 174:436-443.

33. von Graevenitz, A. 1971. Recognition and differential diagnosis of Erwinia herbicola strains isolated in the hospital. Pathol. Microbiol. 37:84-88.

34. Walczak, C. A., and M. I. Krichevsky. 1980. Computer methods for describing groups from binary phenetic data: preliminary summary and editing of data. Int. J. Syst. Bacteriol. 30:615-621.

35. Walczak, C. A., and M. I. Krichevsky. 1980. Computer methods for describing groups from binary phenetic data: modification of numerical taxonomy programs to increase flexibility. Int. J. Syst. Bacteriol. 30:622-626.

36. Walczak, C. A., and M. I. Krichevsky. 1982. Computer-aided selection of efficient identification features and calculation of group descriptors as exemplified by data on Capnocytophaga species. Curr. Microbiol. 7:199-204. 